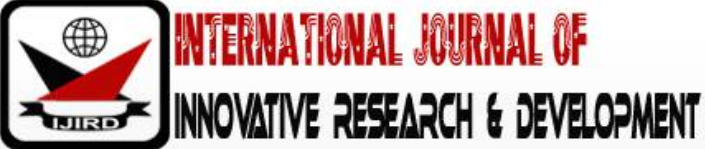

ISSN 2278 - 0211 (Online)

\section{Determinants of Uptake of Intermittent Preventive Treatment for Malaria among Pregnant Women in Suba Sub-County, Homabay County, Kenya}

Kenneth Otieno Omodhi
Postgraduate Student, Department of Public Health and Community, School of Health Sciences,
Jaramogi Oginga Odinga University of Science and Technology, Kenya
Dr. Charles Angira
Lecturer, Department of Public and Community Health \& Development,
Jaramogi Oginga Odinga University of Science and Technology, Kenya
Samson Ong'wen Adoka
Lecturer, Department of Medical Laboratory Sciences,
Maseno University, Private Bag Maseno, Kenya

\begin{abstract}
:
Background: Intermittent preventive treatment of malaria in pregnancy (IPTp) is one of the effective and efficacious interventions recommended for prevention of malaria in pregnancy. The drug of choice is Sulphadoxine-Pyrimethamine (SP) and Kenya adopted the use of IPTp in line with revised 2012 recommendation by WHO.

Method: A cross sectional study was carried out in Suba Sub County to established determinants of uptake of IPTp, from July 2017 to December 2019. The target population was women of reproductive age (15-49 years old) who had live births two years preceding the study and who visited ANC clinic. All health facilities providing ANC services were purposively selected and stratified Systematic random sampling technique was used to sample the mothers who had given birth during the last 24 months from ANC clients register. Quantitative data collection was carried out using questionnaires that were administered to respondents. All mother-child booklets were checked to confirm whether the respondent received IPTp during her last pregnancy.

Results: A total of 382 women who had live births two years prior to the study and who sought ANC services were interviewed. The study revealed that $59.5 \%$ of respondents received three or more doses of IPTp-SP, with $17.2 \%$ receiving at least one dose. Women who attended ANC clinic three or more times had a higher odds of receiving three or more doses of IPTp-SP (OR=1.0), compared with women who attended clinic less than three times (OR=0.04).Women who stayed six or more Km away from health facility were less likely to receive three or more doses of IPTp-SP $(O R=0.21)$. Women who took 30 or more minutes to reach health facility were most likely to receive three or more doses of IPTp -SP (OR=2.93) compared with women who took less than 30 minutes.

Conclusion: The study revealed that majority of pregnant women attended ANC clinic three or more times and not all women interviewed received the recommended three or more doses of IPTp.
\end{abstract}

Keywords: Determinants, malaria, prevention, pregnancy, uptake of IPTp, SP, Kenya

\section{Background Information of the Study}

Malaria still remains one of the leading causes of morbidity and mortality worldwide. Globally, close to 214 million malaria cases with 435,000 deaths have been reported (WHO, 2015). About 91\% of these malaria deaths occur in Sub Saharan Africa. In Sub Saharan Africa, close to 25 million pregnant women are at risk of plasmodium falciparum infection annually and in every four women, one has an evidence of placental infection at the time of giving birth. This shows that women and children are highly vulnerable to malaria infection during pregnancy (Desai et al., 2007). In Africa yearly 10,000 maternal deaths and about 200,000 fetal and infant deaths are attributed to malaria. Malaria in pregnancy is associated to $11.4 \%$ of neonatal deaths and $5.7 \%$ of all infant deaths and these are due to low birthweight, which translate to 100,000 infant deaths (Richard et al., 2001). In low risk zones, episodes of severe malaria significantly associate with stillbirths, spontaneous abortion, premature delivery and maternal death. However, in high-risk areas, women are susceptible to asymptomatic infection, with potential results being maternal anemia and placental parasitaemia. Both situations are conducive for low birth weight and subsequently, infant mortality. Globally, about 50 million women become pregnant each year, of which more than one-half reside in Africa, where the risk of malaria infection is highest (Dellicour, 2007). 
Intermittent Preventive Treatment in pregnancy (IPTp) is one of the key interventions recommended by WHO to be implemented in malaria endemic zones for the prevention of asymptomatic infections among pregnant women, besides the use of insecticide-treated bed nets as well as timely and effective management of clinical malaria and anemia (WHO, 2013). Successful implementation of IPTp reduces the risk of severe maternal anemia by $38 \%$, low birth weight by $43 \%$ and perinatal morbidity by $27 \%$ (Desai et al., 2007). IPTp intervention is routinely provided through existing antenatal (ANC) programs in all health facilities. In this regard, all pregnant women receive the first dose of three tablets (IPTp1), which providers administer under their direct observation at the antenatal care (ANC) facilities within the 16th week of gestation. Subsequently, the second and third doses are given four weeks apart during ANC visit (WHO, 2013). The effectiveness of this intervention and the risk resulting from malaria infection in pregnancy prompted Roll Back Malaria (RBM) Partnership to set a goal of 100\% coverage of IPTp by 2015 (Roll Back Malaria Partnership, 2011). Many countries are far from achieving this goal. Among 23 African countries that were reported on IPTp coverage levels in 2016, an estimated $19 \%$ of eligible pregnant women received the recommended three or more doses of IPTp, compared with $18 \%$ in 2015 and 13\% in 2014, which is still below the recommended coverage by WHO (WHO, 2017).

In Kenya, IPTp administration is in line with the revised recommendations by WHO of 2012 which states that all pregnant women living in malaria-endemic zones should receive sulfadoxine-pyrimethamine (SP) for prevention of malaria during pregnancy in three doses four weeks apart (monthly) during antenatal (ANC) visits. According to (KMIS, 2015), only $37 \%$ of pregnant women received the recommended three dose or more despite utilization of ANC services of 94\%. Kenya has experienced steady upward trend in IPTp coverage from 2007 to 2015, though there is still need to increase IPTp coverage, particularly for three or more based on low coverage. According to (GoK, 2014), only 17\% of pregnant women age 15-49 with a live birth took the recommended two or more doses of SP/Fansidar, which is given during an ANC visit (GoK, 2014). In Bungoma, it was found out that, early ANC attendance enhances the probability of receiving maximum required doses of IPTp. Women starting ANC visits in the second month of pregnancy had about 10.5 times odds of receiving two or more doses of IPTp compared with those initiating ANC visits in the sixth month of pregnancy (Mutulei, 2013).

\section{Method}

\subsection{Background Information of the Study Area}

The study was carried out in Suba Sub County. The Sub County is located along Lake Victoria and it falls within malaria endemic zone. The Sub County had an estimated population of 128,456 with 61,660 males and 66,796 females respectively. Women of reproductive age was estimated to be 30,836 according to projected population from the 2009 census, and estimated pregnancies were 5583. The economic activities in this locality are fishing, farming and business. Suba Sub County had 377 villages with 24,085 households. A total of 31 Health facilities were offering ANC services that is 4 Level 5 Hospitals, 10 Health Centers ,14 Dispensaries and 2 private clinics. SP was being administered as Directly Observed Therapy at the ANC in all health facilities under the supervision of a nurse (Aryal et al., 2018) .

\subsection{Study Design}

This study applied descriptive study design that was cross sectional in nature and the aim of the study was to establish determinants of IPTp uptake among pregnant mothers in Suba Sub-County. This study targeted to gather the required information from mothers who had given birth to live babies in the last 24 months prior to the study.

\subsection{Study Population}

The study population was all 30,836 who comprised women of reproductive age or child bearing age within an age bracket of between (15-49) years who stayed in Suba Sub County (HBC report, 2019).

\subsection{Target Population}

The target population was 8,172 of mothers (15-49) who attended ANC and received at least one dose of IPTp in Suba Sub-County, who had the potential of participating in this study (HBC report, 2019).

\subsection{Sampling Procedure}

All health facilities providing ANC services were purposively selected and stratified Systematic random sampling technique was used to sample the mothers who had given birth during the last 24 months from ANC clients register, then respondents were identified based on proportionate allocation of sample size focusing on health facility data.

\subsection{Research Instrument}

Data was collected using structured and semi-structured questionnaire administered to the respondent by trained research assistants. Data collection variables included; socio-demographic information of the study population such as age, education level, occupation and gestational age at first ANC visit. Beside these, data was also collected on educational factors which included knowledge on IPTp and effects of malaria in pregnancy, and environmental factors such as distance to the nearest facility, transport and accessibility of the facility for ANC services. 


\subsection{Piloting}

All tools for collecting data were pre-tested before use to ascertain validity and reliability. The questionnaires were all translated in local language and the process of data collection was conducted in the same local language (dholuo). Piloting of the tools took place in Lambwe ward of Suba North Sub County.

\subsection{Data Collection Procedure}

Quantitative data collection was carried out using questionnaires that were administered to respondents. All mother -child booklets were checked to confirm whether the respondent received IPTp during her last pregnancy. The respondents were identified from the ANC registers in all health facilities offering ANC services and included to take part in the study based on the sample size per health facility.

\subsection{Data Analysis}

Data was analyzed after entry into the prepared spread sheet using computer software STATA version13.Data analysis was conducted based on specific objectives and with their thematic areas of interests. The descriptive analysis method was used to present the findings of the research in a descriptive nature about the way they occur concerning specific areas of interest of the study. The inferential statistical analysis method was used as the second level of analysis such as, multivariate and regression in order to make more meaning about the problem that was under investigation. These helped in isolating the relationships between and among variables that the study was trying to establish and the implications. The findings of the analyses of this research were tested using chi-square test. The final findings of the analysis were presented in the tables, figures and narrative forms with the specific objective's areas of research.

\section{Results}

\subsection{Sociodemographic Characteristics of Respondents}

This study established different variables of demographic characteristics that are important in identifying determinants of uptake of IPTp among pregnant mothers in Suba Sub County.

\begin{tabular}{|c|c|c|}
\hline \multirow[t]{2}{*}{ Variable } & \multicolumn{2}{|c|}{ Statistics } \\
\hline & $\mathbf{n}$ & $\%$ \\
\hline \multicolumn{3}{|l|}{ Age $N=382$} \\
\hline$[10-14]$ & 2 & 0.5 \\
\hline [15-19] & 29 & 7.6 \\
\hline$[20-24]$ & 146 & 38.2 \\
\hline $25+$ & 205 & 53.7 \\
\hline \multicolumn{3}{|l|}{ Marital Status N=379 } \\
\hline Married & 334 & 88.1 \\
\hline Single & 34 & 9 \\
\hline Divorce & 5 & 1.3 \\
\hline Separated & 6 & 1.6 \\
\hline \multicolumn{3}{|l|}{ Religion $\mathrm{N}=382$} \\
\hline Muslim & 4 & 1 \\
\hline Christian & 372 & 97.4 \\
\hline No religion & 6 & 1.6 \\
\hline \multicolumn{3}{|l|}{ Occupation $\mathrm{N}=373$} \\
\hline Self Employed & 120 & 32.2 \\
\hline Permanent Employment & 5 & 1.3 \\
\hline Not Employed & 103 & 27.6 \\
\hline Farming & 121 & 32.4 \\
\hline Fishing & 24 & 6.4 \\
\hline
\end{tabular}

Table 1: Background Characteristics of Respondents

Table 1revealed that 54\% (205/382) of the respondents were mothers aged 25 years and above, 38\% (146/382) were aged $20-24$ years, $8 \%(29 / 382)$ were aged $15-19$ years and only $0.5 \%(2 / 382)$ were aged $10-14$ years. It also established that $88 \%$ (334/382) of the respondents were mothers who were married, $9 \%(34 / 382)$ single, $1 \%(5 / 382)$ divorced and only $2 \%(6 / 382)$ were separated. This study also determined that $72 \%(372 / 382)$ of the respondents were Christians, 1\% (4/382) were Muslims with only 1\% (6/382) with no religion. The table also shows that 32\% (131/373)7 of the respondents were farmers, 32\% (120/373) self-employed, $28 \%(103 / 373$ not employed, $6 \%(24 / 373)$ fishermen and $1 \%(5 / 373)$ had permanent employment.

\subsection{Socio-Demographic Determinants of IPTp Uptake among Pregnant Women}

The Socio-Demographic determinants are very key variables that influence the uptake of IPTp among pregnant mothers as a method of malaria prevention. 


\begin{tabular}{|c|c|c|c|c|c|c|}
\hline & \multicolumn{2}{|c|}{$\begin{array}{c}\text { Who received } \\
\text { at least } 1 \text { dose of IPTp }\end{array}$} & \multirow[t]{2}{*}{$\begin{array}{l}\text { Chi-Square } \\
\text { P-Value }\end{array}$} & \multicolumn{2}{|c|}{$\begin{array}{c}\text { Who received } \\
3+\text { doses of IPTp }\end{array}$} & \multirow[t]{2}{*}{$\begin{array}{c}\text { Chi-Square } \\
\text { P-Value }\end{array}$} \\
\hline & $\mathrm{n}$ & $\%$ & & $\mathrm{n}$ & $\%$ & \\
\hline Occupation & & & & & & \\
\hline Self Employed & 108 & 31.6 & & 59 & 28.8 & \\
\hline P Employment & 5 & 1.5 & & 4 & 2 & \\
\hline Not Employed & 97 & 28.4 & & 58 & 28.3 & \\
\hline Farming & 113 & 33.0 & & 79 & 38.5 & \\
\hline Fishing & 19 & 5.6 & & 5 & 2.4 & \\
\hline Total & 342 & 100.0 & 0.129 & 205 & 100.0 & 0.001 \\
\hline
\end{tabular}

Table 2: Association between Occupation and Iptp Uptake

Table 2 shows association of occupation and IPTp uptake. The result reveals ( $p$-value $=0.129$ ) for receiving at least one dose of IPTp and (p-value=0.001) for three or more doses of IPTp. Occupation was therefore only significantly associated with uptake of three or more doses of IPTp, while it was not significantly associated with uptake of at least one dose of IPTp.

\begin{tabular}{|c|c|c|c|c|c|c|}
\hline & \multicolumn{2}{|c|}{$\begin{array}{c}\text { Who received } \\
\text { at least 1 dose of IPTp }\end{array}$} & $\begin{array}{c}\text { Chi- } \\
\text { Square } \\
\text { P-Value }\end{array}$ & \multicolumn{2}{c|}{$\begin{array}{c}\text { Who received } \\
\text { 3+ doses of IPTp }\end{array}$} & $\begin{array}{c}\text { Chi-Square } \\
\text { P-Value }\end{array}$ \\
\hline & $\mathrm{n}$ & $\%$ & & $\mathrm{n}$ & $\%$ & $\mathrm{n}$ \\
\hline Marital status & & & & & & \\
\hline Married & 308 & 88.8 & & 187 & 90.8 & \\
\hline Single & 30 & 8.6 & & 15 & 7.3 & \\
\hline Divorce & 3 & 0.9 & & 0 & 0 & \\
\hline Separated & 6 & 1.7 & & 4 & 1.9 & \\
\hline Total & 347 & 100.0 & 0.053 & 206 & 100.0 & 0.044 \\
\hline
\end{tabular}

Table 3: Association between Marital Status and IPTp Uptake

Table 3 illustrates association of marital status and uptake of IPTp. The p-value for association between marital status and receiving both at least one doses of IPTp and three plus doses were ( $p$-value $=0.053$ ) and ( $p$-value $=0.044)$ respectively. The results indicate that marital status was not significantly associated with uptake of at least one dose of IPTp and only significantly associated with uptake of 3 or more doses.

\begin{tabular}{|c|c|c|c|c|c|c|}
\hline & \multicolumn{2}{|c|}{$\begin{array}{c}\text { Who received } \\
\text { at least 1 dose of IPTp }\end{array}$} & $\begin{array}{c}\text { Chi-Square } \\
\text { P-Value }\end{array}$ & \multicolumn{2}{|c|}{$\begin{array}{c}\text { Who received } \\
\mathbf{3}+\text { doses of IPTp }\end{array}$} & $\begin{array}{c}\text { Chi-Square } \\
\text { P-Value }\end{array}$ \\
\hline Religion & $\mathbf{n}$ & $\mathbf{9}$ & & $\mathbf{n}$ & $\mathbf{\%}$ & \\
\hline & & & & & & \\
\hline Muslim & 4 & 1.1 & & 3 & 1.4 & \\
\hline Christian & 341 & 97.4 & & 203 & 97.1 & \\
\hline No religion & 5 & 1.4 & & 3 & 1.4 & \\
\hline Total & 350 & 100.0 & 0.636 & 209 & 100.0 & 0.697 \\
\hline
\end{tabular}

Table 4: Association between Religion and IPTp uptake

Table 4 shows the result of Chi Square analysis of religion as a sociodemographic determinant of uptake of IPTp. The table revealed a $p$-value of ( $p$-value $=0.636)$ and $(p$-value $=0.679)$ for receiving at least one dose and three or more doses respectively. Religion was not significantly associated with receiving of both at least one dose and 3 plus doses of IPTp. 


\begin{tabular}{|c|c|c|c|c|c|c|}
\hline & \multicolumn{2}{|c|}{$\begin{array}{c}\text { Who received at } \\
\text { least } 1 \text { dose of IPTp }\end{array}$} & \multirow[t]{2}{*}{$\begin{array}{c}\text { Chi-Square } \\
\text { P-Value } \\
\end{array}$} & \multicolumn{2}{|c|}{$\begin{array}{c}\text { Who received } \\
3+\text { doses of IPTp }\end{array}$} & \multirow[t]{2}{*}{$\begin{array}{c}\text { Chi-Square } \\
\text { P-Value }\end{array}$} \\
\hline & $\mathbf{n}$ & $\%$ & & $\mathbf{n}$ & $\%$ & \\
\hline \multicolumn{7}{|c|}{$\begin{array}{l}\text { ANC clinic attendance } \\
\text { during last pregnancy }\end{array}$} \\
\hline Yes & 346 & 98.9 & & 206 & 98.6 & \\
\hline No & 4 & 1.1 & & 3 & 1.4 & \\
\hline Total & 350 & 100.00 & 0.000 & 209 & 100.00 & 0.112 \\
\hline \multicolumn{7}{|c|}{$\begin{array}{c}\text { Gestational age at } \\
\text { 1st ANC }\end{array}$} \\
\hline$[1-3]$ & 115 & 33.0 & & 76 & 36.0 & \\
\hline [4-6] & 202 & 58.0 & & 127 & 61.0 & \\
\hline [7-9] & 32 & 9.0 & & 6 & 3.0 & \\
\hline Total & 349 & 100.0 & 0.043 & 209 & 100.0 & 0.000 \\
\hline \multicolumn{7}{|c|}{ At least 3 ANC Visits } \\
\hline Yes & 273 & 78.0 & & 201 & 96.2 & \\
\hline No & 77 & 22.0 & & 8 & 3.8 & \\
\hline Total & 350 & 100.0 & 0.000 & 209 & 100.0 & 0.000 \\
\hline
\end{tabular}

Table 5: ANC Related Determinants and Uptake of Iptp

In table 5 presents ANC related determinants. The table reveals a p-value for ANC clinic attendance in the last pregnancy (p-value=0.000), gestational age at first ANC (p-value $=0.043$ ) and having at least 3 ANC attendance (pvalue $=0.000$ ) for receiving at least one dose of IPTp. The results for receiving three or more doses of IPTp were; ANC clinic attendance in the last pregnancy ( $p$-value $=0.112$ ), gestational age at first ANC ( $p$-value $=0.000)$ and having at least three ANC clinic visits ( $p$-value=0.000). ANC clinic attendance in the last pregnancy, gestational age at first ANC and having at least 3 ANC attendance were significantly associated with receiving at least one dose of IPTp, while gestational age at first ANC and having at least three ANC clinic visits were significantly associated with receiving three or more doses of IPTp.

\begin{tabular}{|c|c|c|c|}
\hline Determinant & Odds & P-value & {$[95 \% \mathrm{CI}]$} \\
\hline \multicolumn{4}{|l|}{ Occupation } \\
\hline Self Employed & Ref & & \\
\hline Permanent Employed & 1 & & \\
\hline Not Employed & 1.33 & 0.366 & $(0.72,2.46)$ \\
\hline \multicolumn{4}{|l|}{ Marital Status } \\
\hline Married & Ref & & \\
\hline Single & 0.95 & 0.912 & $(0.35,2.57)$ \\
\hline Divorce & 1 & & \\
\hline Separated & 1.65 & 0.666 & $(0.17,15.89)$ \\
\hline \multicolumn{4}{|l|}{ Gestation Age at $1^{\text {st }}$ ANC } \\
\hline$[1-3]$ & Ref & & \\
\hline [4-6] & 0.63 & 0.126 & $(0.35,1.14)$ \\
\hline$[7-9]$ & 0.43 & 0.202 & $(0.12,1.58)$ \\
\hline \multicolumn{4}{|l|}{ Attended 3+ ANC visits } \\
\hline Yes & Ref & & \\
\hline $\begin{array}{ll}\text { No } \\
\end{array}$ & 0.04 & 0.000 & $(0.02,0.1)$ \\
\hline \multicolumn{4}{|c|}{$\begin{array}{l}\text { Knowledge on Malaria effect during } \\
\text { pregnancy }\end{array}$} \\
\hline Good & Ref & & \\
\hline Poor & 0.94 & 0.87 & $(0.45,1.96)$ \\
\hline \multicolumn{4}{|l|}{ Malaria information (Nurse) } \\
\hline Yes & Ref & & \\
\hline No & 1.71 & 0.104 & $(0.9,3.26)$ \\
\hline \multicolumn{4}{|l|}{ Malaria information (CHV) } \\
\hline Yes & Ref & & \\
\hline No & 0.88 & 0.687 & $0.46,1.67)$ \\
\hline \multicolumn{4}{|l|}{ Distance in $\mathrm{Km}$} \\
\hline$[0-2]$ & Ref & & \\
\hline$[2-4]$ & 0.65 & 0.215 & $(0.33,1.29)$ \\
\hline [4-6] & 0.51 & 0.105 & $(0.23,1.15)$ \\
\hline$[6+]$ & 0.21 & 0.001 & $(0.08,0.55)$ \\
\hline \multicolumn{4}{|l|}{ Time Taken in Minutes } \\
\hline$[1-10]$ & Ref & & \\
\hline [11-20] & 1.02 & 0.974 & $(0.36,2,89)$ \\
\hline [21-30] & 2.73 & 0.05 & $(1.0,7.51)$ \\
\hline$[30+]$ & 2.93 & 0.032 & $(1.09,7.82)$ \\
\hline
\end{tabular}

Table 6: Logistic Regression Analysis of Determinants of Uptake of 3+ Iptp Doses Compared to Uptake of at Least One Dose of Iptp 
Table 6 presents findings of logistic regression analysis of determinants of uptake of three or more doses of IPTp. It indicates that ANC visits of three or more, distance in Km of six or more, and time of 30 minutes or more taken to reach health facility are positively associated with uptake of three or more doses of IPTp. Women who attended ANC clinic three or more times had a higher odds of receiving three or more doses of IPTp (OR=1.0), compared with women who attended clinic less than three times $(\mathrm{OR}=0.04)$, similarly women who stayed six or more Km away from health facility were less likely to receive three or more doses of IPTp $(\mathrm{OR}=0.21)$ compared to women who stayed less than $6 \mathrm{Km}$ away. The table also indicates that women who took 30 or more minutes to reach health facility were most like to receive three or more doses of IPTp (OR=2.93) compared with women who took less than 30 minutes. Employment, marital status, knowledge on the effects of malaria in pregnancy and sources of information of malaria in pregnancy were not significantly associated with uptake of three and more doses of IPTp.

\section{Discussion}

The current study established that occupation and marital status were significantly associated with uptake of three or more doses of IPTp-SP. Two studies in Tanzania found out that, occupation determined uptake of both minimal and optimal uptake of IPTp-SP (Exavery et al., 2014) and (Kibusi et al., 2015). Financial resources could facilitate pregnant mothers to access ANC services at their convenient time, and this could also help them pay for other charges at health facility. Support from family members could also motivate pregnant women to attend ANC clinic. Discussion of the benefits of attending ANC earlier at the household level for both the pregnant mother and her spouse greatly impact positively to clinic attendance. The result of this study also indicates that marital status was significantly associated with receiving three or more doses of IPTp-SP. Exavery et al in a study in six districts of Tanzania found out that marital status was only significantly associated with partial but not optimal use of IPTp-SP.

This study also found out that gestational age at first ANC was statistically significantly associated with uptake of IPTp-SP .In Bosomtwe, Ghana (Antwi 2010) found out that gestational age at first ANC was not a predictor of receiving two or more doses of IPTp-SP , while in Zimbabwe (Chikwasha et al., 2014) established that gestational age at first ANC was a predictor of IPTp uptake. Starting clinic early makes pregnant mothers start getting information on prevention of malaria in pregnancy early, and this results to visiting clinic regularly on appointment time. The end result is receiving more than one dose of IPTp. Ante Natal Care clinic attendance determines the number of IPTp doses a pregnant woman should get in the entire pregnancy period. The more the number of ANC attendance the more likely is the number of IPTp doses she is to get. The result of this study indicates that three or more ANC visit was significantly associated with uptake of both minimal and maximum doses of IPTp. Selina and Francis in a cross sectional study carried out in private health facilities in Tema Metropolis, Ghana (Amankwah \& F.Anto., 2019) found out that ,more ANC visits are associated with taking more doses of SP

\section{Conclusion}

Occupation, marital status, ANC attendance, gestational age at first ANC, three or more ANC clinic attendance, knowledge on the effects of malaria in pregnancy, source of information on malaria in pregnancy and distance to the nearest health facilities determines uptake of IPTp in Suba Sub County. Despite the significance of these determinants, pregnant women still could not get the required number of doses of IPTp-SP to prevent them from getting malaria in pregnancy and this led to some contacting malaria infection during pregnancy. In order to address these gaps Ministry of health should initiate community engagement and participation in promoting prevention of malaria in pregnancy and early initiation of ANC attendance by all pregnant women. This can be done through the use of community health services by involving all CHVs. Family education by Community Health Volunteers at household level and health education by nurses at the clinic to provide more information on malaria in pregnancy and Health systems through ministry of health in partnership with health partners to organize more community outreaches in hard to reach areas to promote access to ANC services by pregnant women.

\section{Ethical Consideration}

Ethical approval for the study was obtained from the Ethical Committee of JOOUST Re: JOOUST/DVC-RIO/ERC/E2 and approval number 7/13/ERC/10/2019(f), National Commission for Science, Technology and Innovation (NACOSTI) Ref No: 456116 and License No: NACOSTI/P/19/2451, and finally permission was obtained from Homabay County Ministry of Health Ref: MOH/RAVOL3 (008). All participants were given a written informed consent form to fill before the start of data collection. Participation in the study was made voluntary and participants could opt out at any stage of the study.

\section{Abbreviations}

AIDS-Acquired Immune Deficiency Syndromes,

ANC-Ante Natal Care,

DOT-Directly Observed Treatment,

GoK-Government of Kenya,

IPTp-Intermittent Preventive Treatment in Pregnancy,

ITN-Insecticide Treated Net,

KDHS-Kenya Demographic Health Survey,

KMIS-Kenya Malaria Indicator Survey,

KNBS-Kenya National Bureau of Statistics,

MOH -Ministry of Health, 
RBM-Roll Back Malaria,

KNMS-Kenya National Malaria Strategy,

SCMOH-Sub County Medical Officer of Health,

SP-Sulphadoxine-Pyrimethamine,

UNICEF-United Nations Children's Fund,

WHO-World Health Organization.

\section{Author contributions}

The study was put together and designed by KOO and the first author conducted the study in Suba Sub County of Homabay County, carried out data analysis and prepared the manuscript. CA and SOA supervised and gave their technical input during the development of the study and field work and the development of this manuscript.

\section{Disclaimer}

The findings and conclusions presented in this manuscript are those of the authors and do not necessarily reflect the official position of Maseno University

\section{Acknowledgement}

We appreciate and acknowledge all individuals who participated in this study and who made it to succeed, SCMOH Suba North and Suba South and Director of Preventive and Promotive Health, Homabay County.

\section{Funding}

The research was supported from support from friends and workmates

\section{References}

i. Akinleye, S., O. , Falade.C. O, \& Ajayi.I.O. (2009). Knowledge and utilization of intermittent preventive treatment formalaria among pregnant women attending antenatal clinics in primary health care centers in rural southwest, Nigeria: a cross-sectional study. BioMed Central.

ii. Amankwah, S., \& F.Anto. (2019). Factors Associated with Uptake of Intermittent Preventive Treatment of Malaria in Pregnancy: A Cross-Sectional Study in Private Health Facilities in Tema Metropolis, Ghana. Hindawi Journal of Tropical Medicine, 2019.

iii. Antwi , D. G. (2010). Factors influencing the uptake of intermittent preventive treatment of malaria in pregnancy in the Bosomtwe district of Ghana. French Embassy Small Grants programme in the Humanities and Social Sciences.

iv. Arinaitwe, E., et al. (2013). Intermittent Preventive Therapy with Sulfadoxine-Pyrimethamine for Malaria in Pregnancy: A Cross Sectional Study from Tororo, Uganda. PLoS ONE, 8(9).

v. Aryal, K., K., Dangol.R, Gartoulla.P, \& Subedi.G.R. (2018). Health services availability and readiness in seven provinces of Nepal DHS Further Analysis Reports No. 115. Rockville, Maryland, USA: ICF.

vi. Atasige, S. (2014). Factors Influencing the Uptake of Intermittent Preventive Treatment of Malaria among Pregnant Women in the Gushegu District, Nothern Region,Ghana. Masters of Philosophy Degree in Applied Epidemiology and Disease Control-University of Ghana.

vii. Azizi, S., C., et al. (2018). Uptake of intermittent preventive treatment for malaria during pregnancy with Sulphadoxine-Pyrimethamine (IPTp-SP) among postpartum women in Zomba District, Malawi: a cross-sectional study. BMC Pregnancy and Children, 18.

viii. Boateng, E. Y., G.E.Anyormi, J.Otoo., \& D.A.Abaye. (2018). Drivers of intermittent preventive treatment of malaria during pregnancy in Ghana: a generalized linear model with negative binomial approach. Applied Informatics.Springer.

ix. Chikwasha, V., Phiri, I., Chimberengwa, P., Bangure, D., \& Rusakaniko, S. (2014). Predictors of IPTp Uptake among Pregnant Women in the 2010-2011 Zimbabwe Demographic and Health Survey.DHS Working Paper No.112 (Zimbabwe Working Papers No.13). Rockville,Maryland USA:ICF International.,112,1-9.

x. Desai , M., et al. (2007). Epidemiology and burden of malaria in pregnancy. LANCET, 7.

xi. Exavery, A., et al. (2014). Factors affecting uptake of optimal doses of sulphadoxine-pyrimethamine for intermittent preventive treatment of malaria in pregnancy in six districts of Tanzania. Malaria, 13(22).

xii. GoK. (2014). Kenya Demographic and Health Survey.

xiii. Gross, K., et al. (2011). The combined effect of determinants on coverage of intermittent preventive treatment of malaria during pregnancy in the Kilombero Valley, Tanzania. Malaria Journal., 10:140.

xiv. Hajira., et al. (2017). Factors influencing uptake of intermittent preventive treatment of malaria in pregnancy using sulphadoxine pyrimethamine in Sunyani Municipality, Ghana. PanAfrican Medical Journal.

xv. Hill., et al. (2013). Factors Affecting the Delivery, Access, and Use of Interventions to Prevent Malaria in Pregnancy in Sub-Saharan Africa: A Systematic Review and Meta-Analysis. PLoS ONE, 10(7).

xvi. Homabay. (2016-2017). IPTp coverage.

xvii. Ibrahim, H., et al. (2017). Factors Influencing Uptake of Intermittent Preventive Treatment of Malaria in Pregnancy Using Sulphadoxine Pyrimethamine in Sunyani Municipality,Ghana. PanAfrican Medical Journal. 
xviii. Katherine , A., . Tanya ,M,. Pili ,C,. Pasiens ,M,. , \& Hugh , R., . (2008). Timing of intermittent preventive treatment for malaria during pregnancy and the implications of current policy on early uptake in north-east Tanzania. Malaria Journal., 79, 7.

xix. Kibusi , S., M., Kimunai .E, \& S., H. C. (2015). Predictors for uptake of intermittent preventive treatment of malaria in pregnancy (IPTp) in Tanzania. BMC Public Health.

xx. Kibusi., Kimunai E., \& Hines CS. (2015). Predictors for uptake of intermittent preventive treatment of malaria in pregnancy (IPTp) in Tanzania. BMC Public Health, 15(540).

xxi. KMIS. (2015). Kenya Malaria Indicator Survey (KMIS), 2015. National Malaria Control Program.

xxii. Muhumuza, E., Noel .N, Bonny .E.B, Jessica. N, \& K, E. E. (2016). Factors associated with use of malaria control interventions by pregnant women in Buwunga subcounty, Bugiri District. Malaria Journal.

xxiii. Mutulei, A. C. N. (2013). Factors Influencing the Uptake of Intermittent Preventive Treatment for Malaria in Pregnancy: Evidence from Bungoma East District,Kenya. American Journal of Public Health Research, 1(5), 110123.

xxiv. Negera, A, Abelti, G., Bogile, T., Gebrelassie, T., \& Person, R. (2013). An analysis of the trends, differentials, and key proximate determinates of infant and under-five mortality in Ethiopia: Further analysis of the 2000, 2005, and 2011 Demographic and Health Surveys DHS Further Analysis Reports No. 79. Calverton, Maryland, USA: ICF International.

xxv. Onasoga, et al. (2012). Factors influencing utilization of antenatal care services among pregnant women in Ife Central Lga, Osun State Nigeria. Pelagia Research Library, 3(3), 1309-1315.

xxvi. Protas, J., D. Tarimo., \& Moshiro., C. (2016). Determinants of timely uptake of ITN and SP (IPT) and pregnancy time protected against malaria in Bukoba, Tanzania. BioMed Central.

xxvii. Richard, W., S., Bernard, L., N., Monica , E., P. , \& Clara , M. (2001). The Burden of Malaria in Pregnancy in MalariaEndemic Areas. The American Journal of Tropical Medicine and Hygiene, 64(28).

xxviii. Roman, E., et al. (2019). Determinants of uptake of intermittent preventive treatment during pregnancy: a review. Malaria Journal, 18.

xxix. Sangare , L. R., et al. (2010). Determinants of Use of Intermittent Preventive Treatment of Malaria in Pregnancy: Jinja, Uganda. PLoS ONE, 5(11).

xxx. Sikambale, H. H., \& B., K. S. (2013). Factors Influencing Utilization of Intermittent Presumptive Treatment of Malaria (IPTp) Services by Pregnant Women in Sesheke District of Western Province Zambia. Medical Journal of Zambia,, 40(1).

xxxi. Sikambale, C., Halwindi, H., \& Baboo, K. (2013). Factors Influencing Utilization of Intermittent Presumptive Treatment of Malaria (IPTp) Services by Pregnant Women in Sesheke District of Western Province Zambia. Medical Journal of Zambia., 40(1).

xxxii. Susan, F., R., , et al. (2014). Accessibility, availability and utilisation of malaria interventions among women of reproductive age in Kilosa district in central Tanzania. BMC Health Services Research, 14(452), 10.

xxxiii. Theresia, E., I, A., ., William , K., \& Jean, P., D,. (2013). Impact of environment on mosquito response to pyrethroid insecticides: Facts, evidences and prospects. Elsevier-Insect Biochemistry and Molecular Biology, 43.

xxxiv. Thiam, S., Kimotho.V., \& .P., G. (2013). Why are IPTp coverage targets so elusive in Sub-Saharan Africa? A Systematic Review of Health System Barriers. Malaria Journal, 12.

xxxv. UNICEF. (2011). Multiple Indicator Cluster Surveys.

xxxvi. WHO. (2013). WHO policy brief for the implementation of intermittent preventive treatment of malaria in pregnancy using sulfadoxine-pyrimethamine (IPTp-SP).

xxxvii. WHO. (2015). Trends in Maternal Mortality: 1990 to 2015.

xxxviii. WHO. (2016). Malaria Facts sheet 2015.World Health Organization.

xxxix. WHO. (2017). World Malaria Report 2017. 